Special issue of the 2nd International Conference on Computational and Experimental Science and Engineering (ICCESEN 2015)

\title{
Ac Electrical Properties of Polystyrene/Ferroelectric Barium Stannate Titanate $\mathrm{Ba}\left(\mathrm{Ti}_{0.9} \mathrm{Sn}_{0.1}\right) \mathrm{O}_{3}$ Ceramic Composite
}

\author{
M.-A. Al-Akhras*, S. Saq'an and Z. Ghadieh \\ Bio-Medical Physics Laboratory, Department of Physics, Jordan University of Science and Technology (JUST), \\ P.O. Box 3030, Irbid 22110, Jordan
}

\begin{abstract}
A composite of polystyrene (PS)/ferroelectric barium stannate titanate (BST) with different BST concentration was prepared. The dielectric properties were measured using HP low impedance frequency analyzer. It is found that samples behaved as an RC network. Composites become more capacitive than resistive with increasing frequency while resistance decreases with increasing BST ceramic concentration. Temperature effects were monitored throughout the annealing process. It is found that the capacity and the ac conductivity increased after annealing while the bulk resistance decreased. Our results suggest that, the obtained material is expected to be a promising candidate for electronic ceramics. The study provides a better understanding of the relationship among these parameters.
\end{abstract}

DOI: 10.12693/APhysPolA.130.447

PACS/topics: 77.22.Gm, 77.84.Lf, 72.80.Tm

\section{Introduction}

BST is intensively investigated not only for its dielectric properties but also for its potential use in a wide range of applications [1-3]. BST is a binary solid solution system composed of ferroelectric barium titanate and non-ferroelectric barium stannate where both have perovskite structure [3-6]. The development is currently focused on modification of these properties to meet the market demands for specific applications and uses. The material system with some additives like ceramic can have many applications for various purposes. The effect of adding the ceramic to the polymer was studied by comparing the results of the properties with those of neat polymer. Optimizing process route of BST to prepare full composition range solid solution sample and a linear empirical relation between crystal lattice and tin content was proposed by Wei [6] and suggests that this solid solution system is ultimate mutual soluble [6].

Several ac electrical properties with different composite concentrations studies were conducted and found to be altered by composite concentration, temperature, frequency and relaxation time [7-16]. AbuHijleh et al. observed that ac conductivity and dielectric constant increase with increase of mica content in composite [17]. The objective of this research is to study the dielectric properties with annealing effect.

\section{Materials and methods}

In order to examine the effect of adding a ferroelectric ceramic to the polystyrene the barium stannate titanate ceramic $\mathrm{Ba}\left(\mathrm{Ti}_{0.9} \mathrm{Sn}_{0.1} \mathrm{O}_{3}\right)$ samples were prepared

\footnotetext{
*corresponding author; e-mail: alakmoh@just.edu.jo
}

in two steps following the procedure of our previous study $[18,19]$. In brief, $\mathrm{Ba}\left(\mathrm{Ti}_{0.9} \mathrm{Sn}_{0.1} \mathrm{O}_{3}\right)$ was prepared from regent grade $\mathrm{TiO}_{2}(99.995 \%), \mathrm{BaCO}_{3}(99.999 \%)$ and $\mathrm{SnO}_{2}(99.99 \%)$; predetermined amount was weighted out to yield about $10 \mathrm{~g}$ mixture of the composition desired in terms of oxides.

The low frequency impedance analyzer HP4192 is used in ac measurements at room temperatures in the frequency range $10 \mathrm{~Hz}$ to $1 \mathrm{MHz}$, the impedance, phase angle, capacitance and tanloss were recorded and other parameters were calculated before and after annealing. Samples were located in a parallel plate capacitor with a radius of $13 \mathrm{~mm}$. Samples was annealed at $100^{\circ} \mathrm{C}$ for $1 \mathrm{~h}$ in Mittal Enterprises New Delhi hot air oven with temperature controller.

\section{Results and discussions}

The dielectric variables for polystyrene and composites films have been determined using HP 4192A low frequency range Analyzer Bridge in frequency range between $100 \mathrm{~Hz}$ and $1 \mathrm{MHz}$. The measured quantities included impedance $Z$, phase angle $\theta$, sample capacitance $C$ and tanloss data (not shown). The remaining quantities were calculated as a function of frequency and BST concentration. Temperature effect was monitored throughout annealing process.

\subsection{Dependence of electrical properties on the applied electric field frequency}

Figure 1 represents the dependence of dielectric loss on frequency. The addition of other materials could increase or decrease the dielectric loss. For example, adding tin can decrease dielectric loss of BST ceramics at room temperature $[9,20]$. The diffuseness of the phase transition of BST ceramics is enhanced with increasing tin content [21], while adding zirconium to $\mathrm{BaTiO}_{3}$ results in 
lowering the dielectric loss and broadening the dielectric peak near $T_{\mathrm{c}}[22]$. Furthermore, their high values at low frequency start to gradually decrease with increasing frequency indicating low dissipative energy through dipole alignment, and the absence of substantial dipole movement at high frequencies. In addition, this behaviour was observed in all samples as noticed in the dependence of impedance and phase angle on frequency.

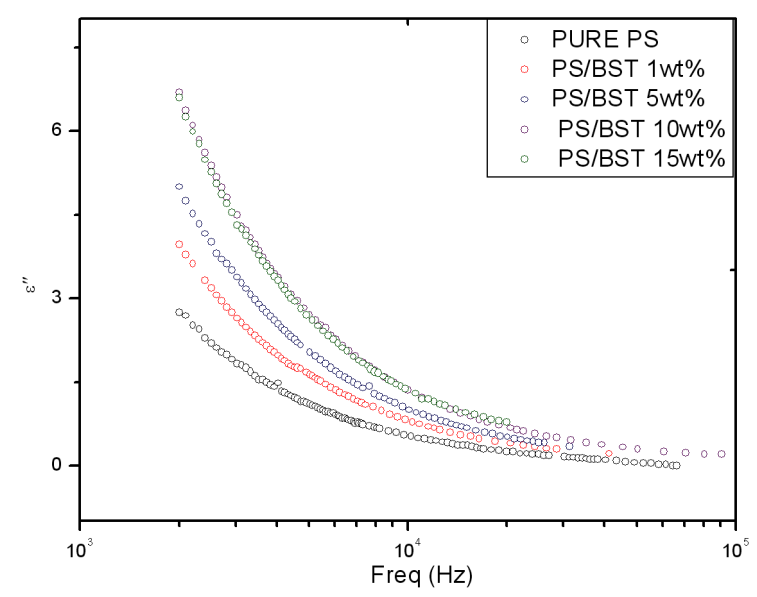

Fig. 1. The dependence of dielectric loss on frequency.

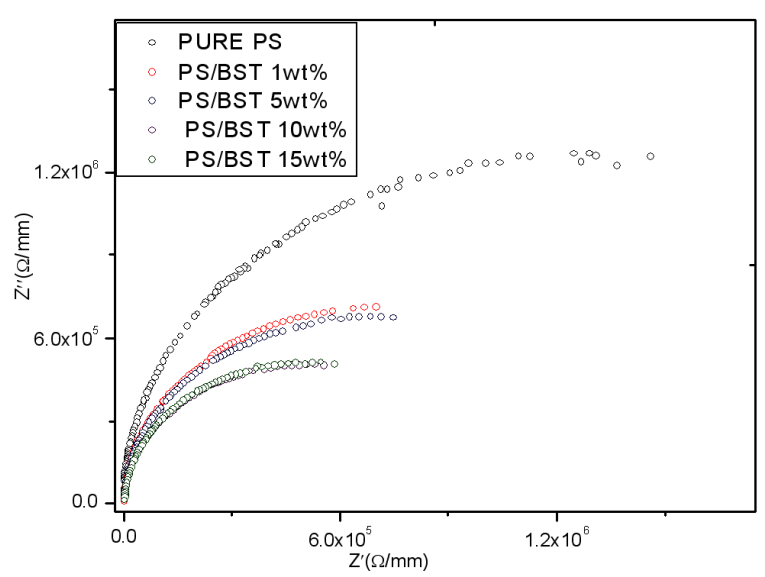

Fig. 2. Cole-Cole plot for all samples.

All samples present one relaxation process and indicate a strong dependence on frequency which is a typical behavior of ionic conduction while reaching a plateau for higher frequencies. At high frequencies a large grains of BST is blocked with the polystyrene matrix, and unable to follow the ac field. Similarly, the imaginary part of impedance shows a single relaxation process. At low frequencies it decreases with increasing frequency which is typical for ionic behavior while at high frequencies its frequency dependence becomes very weak which means that the polystyrene chain is being blocked.

A semicircle obtained in the Cole-Cole plot shown in Fig. 2 indicates that the polystyrene PS/BST composites behaves electrically as an RC circuit network and presents a single relaxation process and was used to investigate the behavior of bulk resistance through its diameter [9]. It is found that there is a clear reduction in bulk resistance from $1.4 \times 10^{6} \Omega / \mathrm{mm}$ for pure PS to $4.8 \times 10^{5}-6.2 \times 10^{5} \Omega / \mathrm{mm}$ for samples of BST concentration $(1-15 \mathrm{wt} \%)$.

\subsection{Dependence of electrical properties on annealing effect}

Ac conductivity, $\sigma_{\mathrm{ac}}$ without annealing increases with increase of frequency, $4.48 \times 10^{-7} \pm 7.24 \times 10^{-9}, 5.67 \times$ $10^{-7} \pm 6.31 \times 10^{-9}, 7.56 \times 10^{-7} \pm 1.12 \times 10^{-8}, 7.43 \times 10^{-7} \pm$ $1.51 \times 10^{-8}$, for the samples PS/BST $1 \mathrm{wt} \%$, PS/BST $5 \mathrm{wt} \% \mathrm{PS} / \mathrm{BST} 10 \mathrm{wt} \% \mathrm{PS} / \mathrm{BST} 15 \mathrm{wt} \%$, respectively, as compared to $3.02 \times 10^{-7} \pm 7.56 \times 10^{-9}(\Omega \mathrm{m})^{-1}$ for pure PS.

This observed conductivity rise can be explained by increase of electronic and ionic mobility of BST. A study of temperature dependence was carried out through annealing process, all samples were annealed for one hour at $100^{\circ} \mathrm{C}$. Comparisons between the electrical properties: impedance, the Cole-Cole plot (bulk resistance), and ac conductivity before and after annealing are illustrated in Figs. 3 and 4. All samples with all concentrations showed small decrease after annealing except sample PS/BST $10 \mathrm{wt} \%$, Figs. 3 and 4.

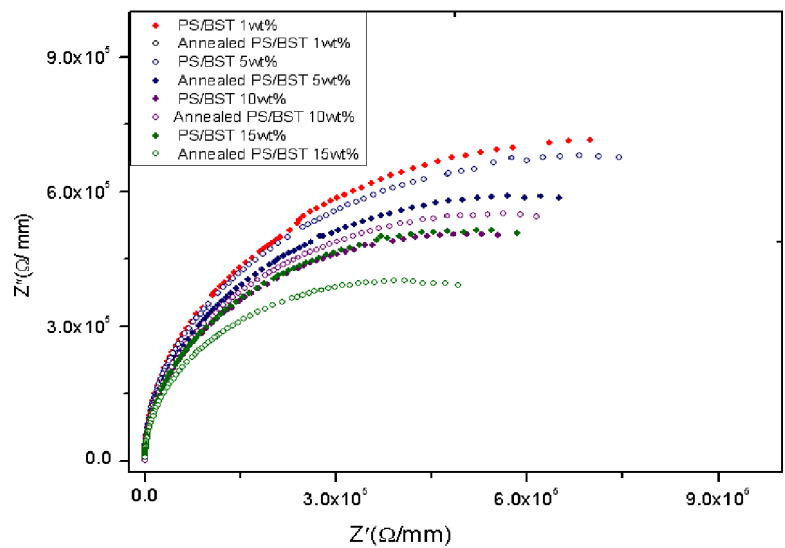

Fig. 3. Cole-Cole plot (bulk resistance) before and after annealing for all composites.

Measurements on dielectric properties of polymer/ferroelectric ceramic composites over broad frequency range from $100 \mathrm{~Hz}$ to $10 \mathrm{GHz}$ and temperature range from 140 to $150{ }^{\circ} \mathrm{C}$ indicate that these properties are strongly dependent on the type of polymer for the same $\mathrm{BaTiO}_{3}$ loading dielectric characteristics of the composites [23]. Furthermore, studying the effects of mixing ferroelectric barium titanate $\left(\mathrm{BaTiO}_{3}\right)$ with ferromagnetic barium hex ferrite $\left(\mathrm{BaFe}_{12} \mathrm{O}_{19}\right)$ showed that the dielectric constant of different concentrations of $\mathrm{BaTiO}_{3}$ decreases as the frequency increases and the dielectric constant increases with temperature until it reaches the 


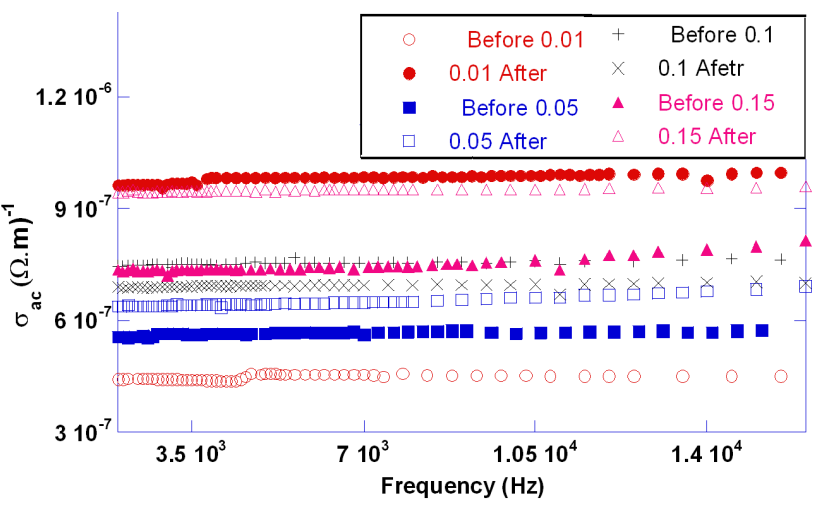

Fig. 4. ac conductivity $\left(\sigma_{\mathrm{ac}}\right)$ vs. frequency before and after annealing for all composites.

Curie temperature and then tends to decrease [24]. The Curie temperature of BTS ceramics decreases with the increase of tin content [21].

In most recent study by Kajtoch et al. similar finding with same percentage was reported when the substitution of lead for barium in the amount of $10 \%$ in the $\mathrm{BP}_{10} \mathrm{TS}_{10}$ ceramics compensates the effect of tin on the temperature of the $\mathrm{PE}-\mathrm{FE}$ phase transition and provides high values of electric permittivity [3]. The occurrence of a $\mathrm{Pb}$ positional fluctuation in the paraelectric phase is typically considered as a formation of polar regions and causes a local distortion of the lattice structure [19], resulting in a change of the electrostatic forces and led to a typical behavior for ferroelectrics with a sharp phase transition. Previous results indicate that $\mathrm{Sn}$ ions can be incorporated in the unit cell while maintaining the perovskite structure of solid solution [21].

\section{Conclusion}

This research covers some of ac electrical properties of polystyrene/ferroelectric barium stannate titanate $\mathrm{Ba}\left(\mathrm{Ti}_{0.9} \mathrm{Sn}_{0.1}\right) \mathrm{O}_{3}$ ceramic composite with concentrations between 1 and $15 \mathrm{wt} \%$. Ac electrical properties was investigated as a function of concentration, frequency, and annealing effect. Parameters such as phase angle, impedance, dielectric constant were measured. Our measurements show that the impedance and the dielectric loss decrease as BST concentration, frequency and temperature (annealing) were increased. The study provides a better understanding of the relationship among these parameters. Our results also show that the phase angle is negative and approaches $-90^{\circ}$ at high frequencies, an indication that samples behave as RC networks. They become more capacitive than resistive with increase of frequency while the phase angle, dielectric constant and capacitance are independent of frequency and BST concentration, before or after annealing. On the other hand, the real and imaginary parts of impedance exhibit a single relaxation peak and one semicircle.

\section{Acknowledgments}

The authors would like to thanks Prof. A. Zihlif from Jordan University and Dr. Khaled Abu Elrub for their great useful discussion. This work was supported by a grant from the Deanship of Scientific Research at Jordan University of Science and Technology (JUST) No. $112 / 2009$.

\section{References}

[1] D. Lisjak, M. Drofenik, D. Kolar, J. Mater. Res. 15, 417 (2000).

[2] M. Kumar, A. Garg, R. Kumar, M.C. Bhatnagar, Physica B 403, 1819 (2008).

[3] C. Kajtoch, W. Bąk, B. Garbarz-Glos, Condens. Matter Phys. 16, 31702 (2013).

[4] H. Takeda, W. Aoto, T. Shiosaki, Appl. Phys. Lett. 87, 102104 (2005).

[5] T. Shimada, K. Touji, Y. Katsuyama, H. Takeda, T. Shiosaki, J. Eur. Ceram. Soc. 27, 3877 (2007).

[6] X. Wei, X. Yao, Mater. Sci. Eng. 137, 184 (2007).

[7] M. Abd El-Hadi, S. Saq'an, A. Zihlif, Adv. Perform. Mater. 23, 152 (2008).

[8] S. Saq'an, A. Ayesh, A. Zihlif, Opt. Mater. 24, 629 (2004).

[9] S. Saq'an, A. Ayesh, A. Zihlif, G. Ragosta, Polym. Test. 23, 739 (2004).

[10] S. Aziz, M. El-Mallah, Int. J. Polym. Mater. 54, 1157 (2005).

[11] K. Nairn, A. Best, P. Newman, D. Mac Farance, M. Farsyth, Solid State Ion. 12, 115 (1999).

[12] M. Altaf, M.S. Choudary, J. Res. Sci. 17, 201 (2006).

[13] S. Ebrahim, A. Kashyout, M. Soliman, Curr. Appl. Phys. 9, 448 (2009).

[14] P. Singh, A. Chandra, J. Phys. D Appl. Phys. 36 , L93 (2003).

[15] S. Saq'an, A. Zihlif, G. Ragosta, J. Thermoplast. Comp. Mater. 21, 457 (2008).

[16] M.I. Mohammed, A. Abdrabo, E. Mahmoud, Egypt. J. Sol. 25, 49 (2002).

[17] M. AbuHijleh, Y. Al-Ramadeen, A. Zihlif, Int. J. Polym. Mater. 46, 377 (2000).

[18] M.-A.H. Al-Akhras, S. Al Saq'an, Z. Ghadieh, AIP Conf. Proc. 1569, 23 (2103).

[19] M.-A.H. Al-Akhras, S. Al Saq'an, Z. Ghadieh, in: 2nd Int. Congress on Energy Efficiency and Energy Related Materials (ENEFM2014), Eds.: A.Y. Oral, Z.B. Bahsi Oral, M. Ozer, Springer Proc. in Energy, Springer International Publishing 2015, p. 335.

[20] A. Abu-Jamous, A.M. Zihlif, Physica B 405, 2762 (2010).

[21] Wei Cai, Yize Fan, Jiacheng Gao, Chunlin Fu, Xiaoling Deng, J. Mater. Sci. 22, 265 (2011).

[22] Z. Yu, C. Ang, R.Y. Guo, A.S. Bhalla, J. Appl. Phys. 92, 2655 (2002).

[23] R. Popielarz, C.K. Chiang, R. Nozak, J. Obzzut, Macromolecules 34, 5910 (2001).

[24] A. Eshtayyah, M.Sc. Thesis, Al Al-Bayt University, Jordan 2006. 